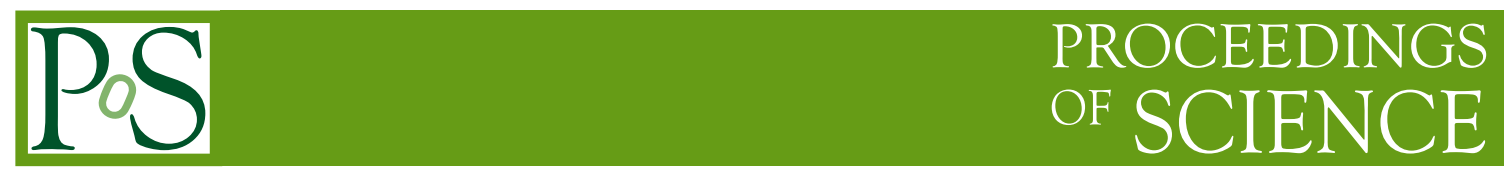

\title{
The anisotropy of cosmic rays observed by the Tibet air shower array and muon detector array
}

M. Amenomori, ${ }^{1}$ Y.-W. Bao, ${ }^{2}$ X. J. Bi, ${ }^{3}$ D. Chen, ${ }^{4}$ T. L. Chen, ${ }^{5}$ W. Y. Chen, ${ }^{3}$ Xu Chen, ${ }^{3}$ Y. Chen, ${ }^{2}$ Cirennima, ${ }^{5}$ S. W. Cui, ${ }^{7}$ Danzengluobu, ${ }^{5}$ L. K. Ding, ${ }^{3}$ J. H. Fang, ${ }^{3,6}$ K. Fang, ${ }^{3}$ C. F. Feng, ${ }^{8}$ Zhaoyang Feng, ${ }^{3}$ Z. Y. Feng, ${ }^{9}$ Qi Gao, ${ }^{5}$ Q. B. Gou, ${ }^{3}$ Y. Y. Guo, ${ }^{3}$ Y. Q. Guo, ${ }^{3}$ H. H. He,${ }^{3}$ Z. T. He, ${ }^{7}$ K. Hibino, ${ }^{10}$ N. Hotta, ${ }^{11}$ Haibing Hu, ${ }^{5}$ H. B. Hu, ${ }^{3}$ J. Huang, ${ }^{3}$ H. Y. Jia, ${ }^{9}$ L.Jiang, ${ }^{3}$ H.-B. Jin, ${ }^{4}$ F. Kajino, ${ }^{12}$ K. Kasahara, ${ }^{13}$ Y. Katayose,${ }^{14}$

C. Kato, ${ }^{15}$ S. Kato, ${ }^{16}$ K. Kawata, ${ }^{16}$ W. Kihara, ${ }^{15}$ Y. Ko,,${ }^{15}$ M. Kozai, ${ }^{17}$ Labaciren, ${ }^{5}$ G. M. Le, ${ }^{18}$ A. F. Li, ${ }^{19,8,3}$ H. J. Li, ${ }^{5}$ W. J. Li, ${ }^{3,9}$ Y.-H. Lin, ${ }^{3,6}$ B. Liu, ${ }^{2}$ C. Liu, ${ }^{3}$ J. S. Liu, ${ }^{3}$ M. Y. Liu, ${ }^{5}$ W. Liu, ${ }^{3}$ Y.-Q. Lou, ${ }^{20}$ H.Lu, ${ }^{3}$ X. R. Meng, ${ }^{5}$ H. Mitsui, ${ }^{14}$ K. Munakata, ${ }^{15}$ H. Nakada, ${ }^{14}$ Y. Nakamura $*{ }^{3}$ H. Nanjo, ${ }^{1}$ M. Nishizawa, ${ }^{21}$ M. Ohnishi, ${ }^{16}$ T. Ohura,,${ }^{14}$ S. Ozawa, ${ }^{22}$ X. L. Qian, ${ }^{23}$ X. B. Qu, ${ }^{24}$ T. Saito, ${ }^{25}$ M. Sakata, ${ }^{12}$ T. K. Sako, ${ }^{16}$ Y. Sengoku, ${ }^{14}$ J. Shao, ${ }^{3,8}$ M. Shibata, ${ }^{14}$ A. Shiomi,${ }^{26}$ H. Sugimoto, ${ }^{27}$ W. Takano,,${ }^{10}$ M. Takita, ${ }^{16}$ Y. H. Tan, ${ }^{3}$ N. Tateyama, ${ }^{10}$ S. Torii, ${ }^{28}$ H. Tsuchiya, ${ }^{29}$ S. Udo, ${ }^{10}$ H. Wang, ${ }^{3}$ H. R. Wu, ${ }^{3}$ L. Xue,${ }^{8}$ K. Yagisawa, ${ }^{14}$ Y. Yamamoto, ${ }^{12}$ Z. Yang, ${ }^{3}$ Y. Yokoe,,${ }^{16}$ A. F. Yuan, ${ }^{5}$

L. M. Zhai, ${ }^{4}$ H. M. Zhang, ${ }^{3}$ J. L. Zhang, ${ }^{3}$ X. Zhang, ${ }^{2}$ X. Y. Zhang, ${ }^{8}$ Y. Zhang, ${ }^{3}$ Yi Zhang, ${ }^{3}$ Ying Zhang, ${ }^{3}$ S. P. Zhao ${ }^{3}$ Zhaxisangzhu, ${ }^{5}$ and X. X. Zhou ${ }^{9}$ (The Tibet AS $\gamma$ Collaboration) 


\author{
${ }^{1}$ Department of Physics, Hirosaki University, Hirosaki 036-8561, Japan \\ ${ }^{2}$ School of Astronomy and Space Science, Nanjing University, Nanjing 210093, China \\ ${ }^{3}$ Key Laboratory of Particle Astrophysics, Institute of High Energy Physics, Chinese Academy of Sciences, \\ Beijing 100049, China \\ ${ }^{4}$ National Astronomical Observatories, Chinese Academy of Sciences, Beijing 100012, China \\ ${ }^{5}$ Physics Department of Science School, Tibet University, Lhasa 850000, China \\ ${ }^{6}$ University of Chinese Academy of Sciences, Beijing 100049, China \\ ${ }^{7}$ Department of Physics, Hebei Normal University, Shijiazhuang 050016, China \\ ${ }^{8}$ Department of Physics, Shandong University, Jinan 250100, China \\ ${ }^{9}$ Institute of Modern Physics, SouthWest Jiaotong University, Chengdu 610031, China \\ ${ }^{10}$ Faculty of Engineering, Kanagawa University, Yokohama 221-8686, Japan \\ ${ }^{11}$ Utsunomiya University, Utsunomiya 321-8505, Japan \\ ${ }^{12}$ Department of Physics, Konan University, Kobe 658-8501, Japan \\ ${ }^{13}$ Shibaura Institute of Technology, Saitama 337-8570, Japan \\ ${ }^{14}$ Faculty of Engineering, Yokohama National University, Yokohama 240-8501, Japan \\ ${ }^{15}$ Department of Physics, Shinshu University, Matsumoto 390-8621, Japan \\ ${ }^{16}$ Institute for Cosmic Ray Research, University of Tokyo, Kashiwa 277-8582, Japan \\ ${ }^{17}$ Institute of Space and Astronautical Science, Japan Aerospace Exploration Agency (ISAS/JAXA), \\ Sagamihara 252-5210, Japan \\ ${ }^{18}$ National Center for Space Weather, China Meteorological Administration, Beijing 100081, China \\ ${ }^{19}$ School of Information Science and Engineering, Shandong Agriculture University, Taian 271018, China \\ ${ }^{20}$ Physics Department, Astronomy Department and Tsinghua Center for Astrophysics, Tsinghua-National \\ Astronomical Observatories of China joint Research Center for Astrophysics, Tsinghua University, Beijing \\ 100084, China \\ ${ }^{21}$ National Institute of Informatics, Tokyo 101-8430, Japan \\ ${ }^{22}$ Advanced ICT Research Institute, National Institute of Information and Communication Technology, \\ Koganei 184-8795, Japan \\ ${ }^{23}$ Department of Mechanical and Electrical Engineering, Shandong Management University, Jinan \\ 250357, China \\ ${ }^{24}$ College of Science, China University of Petroleum, Qingdao, 266555, China \\ ${ }^{25}$ Tokyo Metropolitan College of Industrial Technology, Tokyo 116-8523, Japan \\ ${ }^{26}$ College of Industrial Technology, Nihon University, Narashino 275-8576, Japan \\ ${ }^{27}$ Shonan Institute of Technology, Fujisawa 251-8511, Japan \\ ${ }^{28}$ Research Institute for Science and Engineering, Waseda University, Tokyo 169-8555, Japan \\ ${ }^{29}$ Japan Atomic Energy Agency, Tokai-mura 319-1195, Japan
}

\title{
E-mail: nyoshiakidihep.ac.cn
}

In the TeV energy region, the anisotropy of cosmic ray intensity was observed by some air shower experiments, like Tibet ASgamma, ARGO, HAWC, and so on. But, our previous results are contaminated with the gamma rays and electrons/positrons, and it is difficult to discriminate them with only the plastic scintillation air shower detector. Tibet ASgamma has installed underground muon detector(MD) in 2014. With MD, Tibet ASgamma became capable of discriminating two type primaries in shower events, gamma ray like (due to primary gamma rays, electrons and positron) and cosmic ray like, and to observe the anisotropies of cosmic rays and gamma rays/electrons/positrons, separately. In this work, we will report the result of the anisotropies observed by the Tibet air shower array and muon detector array.

36th International Cosmic Ray Conference -ICRC2019-

July 24th - August 1st, 2019

Madison, WI, U.S.A. 


\section{Acknowledgments}

The collaborative experiment of the Tibet Air Shower Arrays has been conducted under the auspices of the Ministry of Science and Technology of China and the Ministry of Foreign Affairs of Japan. This work was supported in part by a Grant-in-Aid for Scientific Research on Priority Areas from the Ministry of Education, Culture, Sports, Science, and Technology, by Grants-in-Aid for Science Research from the Japan Society for the Promotion of Science in Japan. This work is supported by the National Key R\&D Program of China (No.2016YFE0125500). This work is supported by the Grants from the National Natural Science Foundation of China (Nos.11533007,11673041 and 11873065). This work is supported by the Key Laboratory of Particle Astrophysics, Institute of High Energy Physics, CAS. This work is supported by the joint research program of the Institute for Cosmic Ray Research (ICRR), the University of Tokyo. Y. Nakamura is supported by the CAS President's International Fellowship Initiative (PIFI).

* Speaker. 